[0212-7199 (2005) 22: 8; pp 379-382] ANALES DE MEDICINA INTERNA Copyright $@ 2005$ ARAN EDICIONES, S.L.

AN. MED. INTERNA (Madrid) Vol. 22, N. ${ }^{\circ} 8$, pp. 379-382, 2005

\title{
Meningoencefalitis por Listeria en el lupus
}

\author{
A. LÓPEZ MONTES, E. ANDRÉS MOMPEÁN, M. MARTÍNEZ VILLAESCUSA, \\ A. HERNÁNDEZ BELMONTE', F. MATEOS RODRÍGUEZ'1, L. ABAD ORTIZ², \\ J. PÉREZ MARTÍNEZ, E. LÓPEZ RUBIO, C. GÓMEZ ROLDÁN \\ Sección de Nefrología y de Enfermedades Infecciosas ${ }^{1}$. Servicio de Radiología ${ }^{2}$. Complejo \\ Hospitalario Universitario. Albacete
}

MENINGOENCEPHALITIS BY LISTERIA IN THE LUPUS DISEASE

\section{RESUMEN}

Presentamos un caso de una paciente con nefropatía lúpica de 20 años de evolución en tratamiento con esteroides que desarrolló una meningoencefalitis asociada a bacteriemia por Listeria monocytogenes. La paciente recibió tratamiento antibiótico con ampicilina y gentamicina durante 6 semanas con excelentes resultados. La infección por Listeria monocytogenes afecta predominantemente a pacientes con cierto grado de inmunosupresión, como pacientes con lupus eritematoso sistémico, con una mortalidad alrededor del $30 \%$.

PALABRAS CLAVE: Lupus. Meningoencefalitis. Bacteriemia. Listeria monocytogenes.

\begin{abstract}
We present a patient with lupus nephropathy of 20 years of evolution in treatment with oral steroids who developed a meningoencephalitis associated to bacteraemia by Listeria monocytogenes. The patient was treated successfully with gentamicin and ampicillin for 6 weeks. Infection by Listeria monocytogenes occurs more frequently in individuals with some form of immunedeficiency like lupus disease, with a mortality around $30 \%$.
\end{abstract}

KEY WORDS: Lupus. Meningoencephalitis. Bacteriemia. Listeria monocytogenes.

López Montes A, Andrés Mompeán E, Martínez Villaescusa M, Hernández Belmonte A, Mateos Rodríguez F, Abad Ortiz L, Pérez Martínez J, López Rubio E, Gómez Roldán C. Meningoencefalitis por Listeria en el lupus. An Med Interna (Madrid) 2005; 22: 379-382.

\section{INTRODUCCIÓN}

La infección por Listeria monocytogenes es relativamente frecuente en los recién nacidos, siendo su aparición en la población adulta rara. Afecta principalmente a pacientes con ciertas formas de inmunosupresión, bien sea adquirida por enfermedad o por diferentes tratamientos $(1,2)$.

La infección por Listeria en pacientes con Lupus eritematoso sistémico (LES) es extremadamente rara. Aun así, el LES es considerado factor de riesgo de infección por Listeria monocytogenes (3). Publicamos un caso de una paciente con nefropatía lúpica de 20 años de evolución con importante grado de inmunosupresión que desarrolló una meningoencefalitis asociada a bacteriemia por Listeria monocytogenes.

\section{CASO APORTADO}

Paciente de 54 años diagnosticada desde 1983 de LES con afectación renal (glomerulonefritis lúpica proliferativa segmentaria y focal), cutánea y articular. Durante la progresión de su enfermedad había recibido esteroides orales a diferentes dosis en función de la sintomatología y los marcadores inmunológicos, y 2 ciclos de ciclofosfamida intravenosa, tras 2 brotes de proteinuria y microhematuria. Como complicación había presentado algún episodio de leucopenia, que obligó al cese del tratamiento en alguna ocasión. Tras 19 años de tratamiento con esteroides orales, y permaneciendo a dosis bajas, se decide su retirada (Tablas I y II). Cuatro meses antes del ingreso y 15 meses tras la retirada de esteroides presentó un nuevo brote de nefritis lúpica con proteinuria y microhematuria, que fue tratado con esteroides orales y $750 \mathrm{mgr}$ de ciclofosfamida ( 2 bolos).

La paciente acudió al Servicio de Urgencias de nuestro hospital por fiebre alta $\left(39^{\circ} \mathrm{C}\right)$ con escalofríos, disminución del nivel de conciencia y bradipsiquia de 2-3 horas de evolución. A la exploración llamaba la atención una ligera rigidez de nuca, siendo el resto de la exploración anodina, con un soplo sistólico mitral que ya era conocido. En los datos de laboratorio presentó: sangre: hemoglobina 10,4 $\mathrm{g} / \mathrm{dL}$, hematocrito $29 \%$, Leucocitos $10,35 \times 10^{3} / \mathrm{mcl}$, neutrófilos $7,70 \mathrm{x}$ $10^{3} / \mathrm{mcl}$, plaquetas $198 \times 10^{3} / \mathrm{mcl}$, creatinina $1 \mathrm{mg} / \mathrm{dL}$, urea $78 \mathrm{mg} / \mathrm{dL}$, sodio $138 \mathrm{mEq} / \mathrm{L}$, potasio $4,5 \mathrm{mEq} / \mathrm{L}$, glucosa $98 \mathrm{mg} / \mathrm{dL}$, proteínas totales 6,3 g/dL. En líquido cefalorraquídeo (LCR): leucocitos 196, (95\% mononucleares), glucosa $30 \mathrm{mg} / \mathrm{dL}$, proteínas 1,6 g/dL. En las

Trabajo aceptado: 2 de abril de 2005

Correspondencia: Aurora López Montes. Sección de Nefrología. Complejo Hospitalario Universitario de Albacete. C/ Hnos. Falcó, 37.02006 Albacete. e-mail: auroralmontes@hotmail.com 


\begin{tabular}{|c|c|c|c|c|c|c|c|c|}
\hline \multicolumn{9}{|c|}{ TABLA I } \\
\hline \multicolumn{9}{|c|}{ TRATAMIENTO DEL LES } \\
\hline Fecha & Oct /83 & $\begin{array}{l}\text { Sept /84- } \\
\text { Mayo /90 }\end{array}$ & Mayo /90 & 1992 & Julio 97 & Dic 98 & Agosto/02 & Nov/03 \\
\hline $\begin{array}{l}\text { Ciclofosfamida } \\
\text { (CFC) mg }\end{array}$ & & & $\begin{array}{c}\text { CFC } 1000 \\
\text { (5 bolos) } \\
\text { leucopenia }\end{array}$ & & & $\begin{array}{l}\text { CFC } 1000 \\
\text { (6 bolos) } \\
\text { leucopenia }\end{array}$ & & $\begin{array}{c}\text { CFC } 750 \\
\text { (2 bolos) } \\
\text { leucopenia }\end{array}$ \\
\hline Prednisona (mg) & 60 & $15-2,5$ & $\begin{array}{c}15 \\
\text { glaucoma y } \\
\text { cataratas }\end{array}$ & 10 & $\begin{array}{c}30 \mathrm{mg} \\
\text { osteoporosis } \\
\text { dolor óseo }\end{array}$ & $15 \mathrm{mg}$ & retirada & 60 \\
\hline
\end{tabular}

TABLA II

DATOS ANALÍTICOS DE LA EVOLUCIÓN DEL LES

\begin{tabular}{lccccccccc}
\hline Fecha & Oct 1983 & Sept 1984 & Mayo 1990 & Dic 1992 & Julio 1997 & Dic 1998 & Dic 1999 & Agos 2002 & Nov 2003 \\
\hline ANA & $1 / 640$ & NEG & $1 / 640$ & $1 / 80$ & $1 / 80$ & $1 / 80$ & $1 / 40$ & $1 / 40$ & $1 / 160$ \\
Proteinuria g/ 24h & 1 & 0,2 & 2,2 & 0,15 & 1,6 & 3,3 & 0,2 & 0,05 & 4,3 \\
Hematuria & & & & & & & & & \\
Hematíes/campo & $40-50$ & 25 & 50 & 25 & 100 & 100 & 5 & - & 100 \\
Creatinina mgr/dL & 0,75 & 0,6 & 0,8 & 0,7 & 0,9 & 0,8 & 0,9 & 0,7 & 0,9 \\
\hline
\end{tabular}

exploraciones complementarias presentaba: tomografía computerizada sin contraste: hipodensidad en el lóbulo temporal; resonancia magnética nuclear: áreas de alteración de intensidad de señal de resonancia difusa en la porción central y media del lóbulo temporal derecho, no visualizándose imagen de colección. Hallazgos compatibles con encefalitis a dicho nivel (Fig. 1A); electroencefalograma: actividad

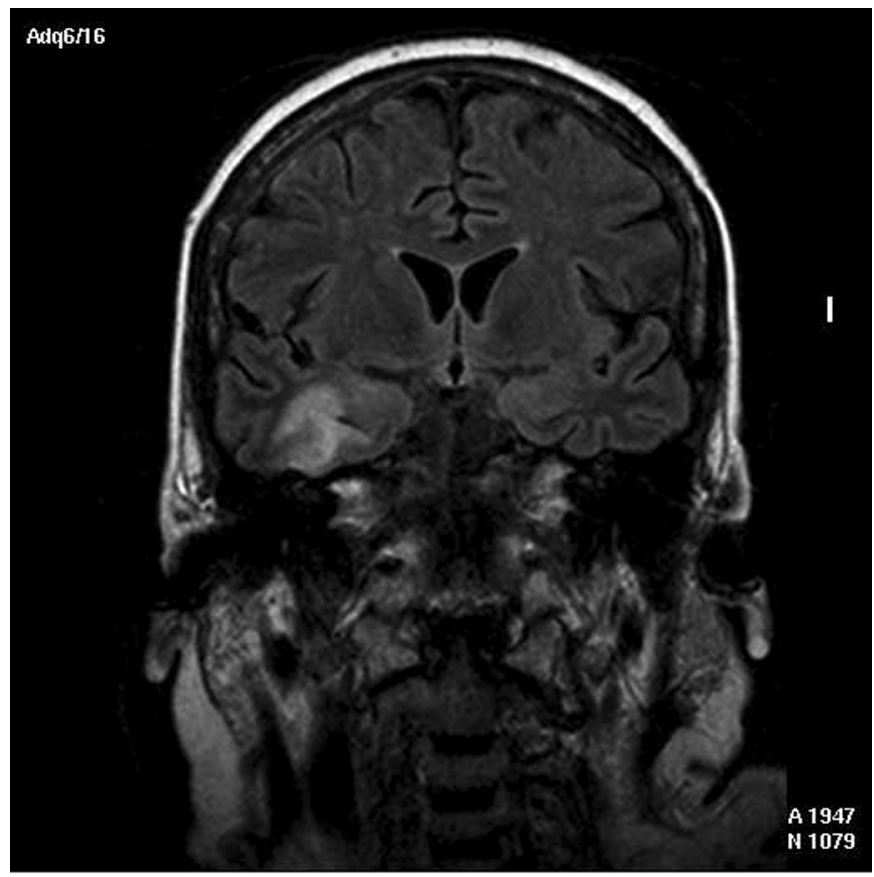

Fig. 1A. RMN: áreas de alteración de intensidad de señal de resonancia difusa en la porción central y media del lóbulo temporal derecho. bioeléctrica cerebral globalmente lentificada y desestructurada. Sobre dicha actividad destaca la presencia de ondas lentas theta-delta irregular, de mayor amplitud a la región homóloga contralateral, de localización centro-temporal derecha; radiografía de tórax normal. Ante la sospecha de meningoencefalitis herpética se comenzó tratamiento con aciclovir intravenosos $600 \mathrm{mg}$ cada 8 horas y ampicilina intravenosa $2 \mathrm{~g}$ cada 4 horas para cubrir otras posibilidades. A las 18 horas de

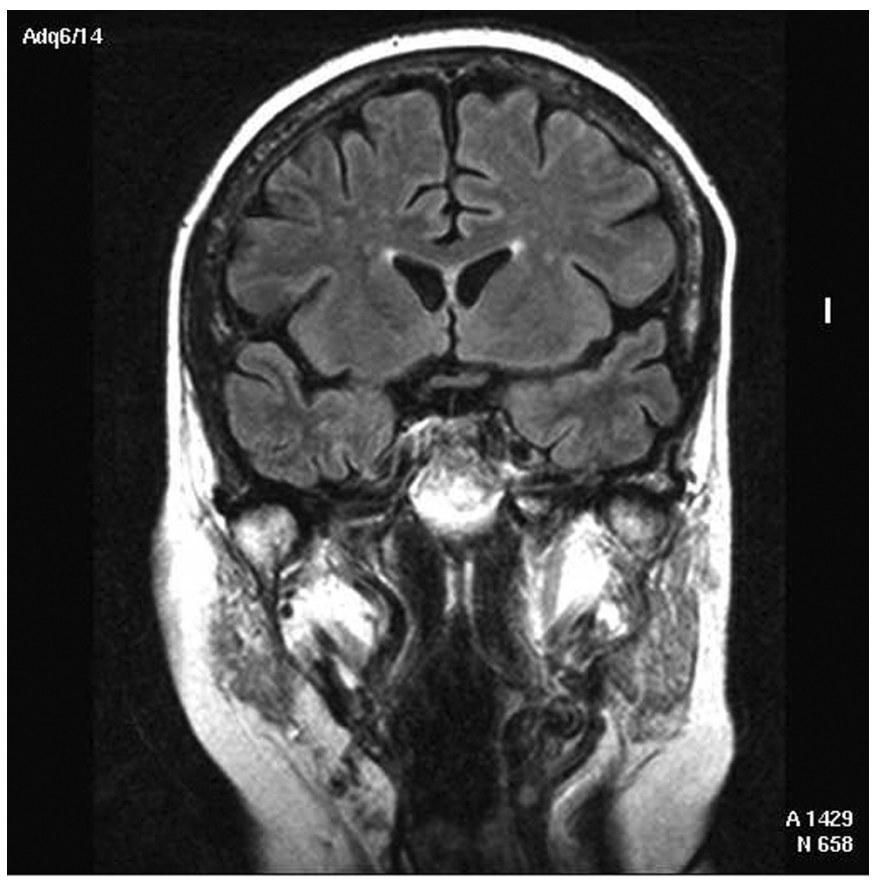

Fig. 1B. RMN tras el tratamiento. Desaparición de las lesiones. 
ingreso se informó del crecimiento de bacilos gram-positivos en sangre, por lo que se asoció gentamicina intravenosa $200 \mathrm{mg}$ en monodosis con control farmacológico (pico $5-12 \mathrm{mcg} / \mathrm{mL}$ ) y se retiró el aciclovir. Los cultivos en sangre y en LCR fueron positivos para Listeria monocytogenes sensible a trimetropin-sulfametoxazol, eritromicina, gentamicina, ampicilina, amoxicilina y vancomicina, y resistente a cefotaxima. La paciente recibió tratamiento con ampicilina durante 6 semanas y gentamicina durante 2 semanas, desapareciendo la sintomatología por completo al tercer día, con mejoría progresiva de los datos de infección en LCR (Tabla III) y desaparición de las alteraciones en las pruebas de imagen (Fig. 1B).

TABLA III

DATOS DEL LÍQUIDO CEFALORRAQUÍDEO

\begin{tabular}{lcccc}
\hline & Leucocitos & Mononucleares & $\begin{array}{c}\text { Glucosa } \\
\mathrm{mg} / \mathrm{dL}\end{array}$ & $\begin{array}{c}\text { Proteínas } \\
\mathrm{g} / \mathrm{L}\end{array}$ \\
\hline Ingreso & 196 & $95 \%$ & 30 & 1,6 \\
10 días & 72 & $90 \%$ & 30 & 2,5 \\
40 días & 15 & & 79 & 5 \\
\hline
\end{tabular}

\section{DISCUSIÓN}

La meningoencefalitis por Listeria monocytogenes es cuadro grave muy infrecuente en nuestro medio. Afecta principalmente a recién nacidos y a pacientes con cierto grado de inmunosupresión, embarazadas, ancianos, pacientes cirróticos, con insuficiencia renal o en diálisis, enfermedades del tejido conectivo, como el lupus, pacientes con cáncer, con síndrome de inmunodeficiencia adquirida, trasplantados, pacientes en tratamiento con corticoides y diabéticos $(1,2)$. También se ha llamado la atención sobre el aumento de la frecuencia de la listeremia en personas sanas (4). Se prevé que la incidencia aumente en la edad adulta relacionado con el progresivo envejecimiento de la población y por el incremento y mayor esperanza de vida de los pacientes inmunodeprimidos (5). La Listeria monocytogenes es un bacilo grampositivo no ácido alcohol resistente, anaerobio facultativo de distribución universal en el medio ambiente, presente en el intestino de mamíferos no humanos, aves, crustáceos y arácnidos. Aunque, el 1$5 \%$ de los humanos son portadores fecales asintomáticos de Listeria monocytogenes.

Se ha implicado al tracto gastrointestinal como la puerta de entrada en diversas ocasiones. La infección se suele producir por la ingestión de productos lácteos, carnes poco cocinadas, perritos calientes, quesos y verduras crudas contaminadas. La Listeria puede multiplicarse entre $1{ }^{\circ} \mathrm{C}$ y 45 ${ }^{\circ} \mathrm{C}$, tiene capacidad de replicarse en concentraciones relativamente altas de sal y crecer en temperaturas de refrigeración.

La listeremia es una enfermedad con manifestaciones muy diversas que incluyen endocarditis, endoftalmitis, peritonitis, colecistitis y abscesos viscerales, linfadenitis, empiema, así cómo infecciones de prótesis articulares y sistemas de derivación ventrículo-peritoneal $(6,7)$. No obstante las manifestaciones más frecuentes con gran diferencia son la sepsis primaria y la meningoencefalitis (8). El porcentaje de enfermos con meningitis y hemocultivos positivos oscila entre un 30 y $86 \%$, presentando estos una mortalidad superior a la de aquellos con hemocultivo negativo (9). El curso clínico de pacientes con meningoncefalitis por Listeria suele ser muy severo y en ocasiones fatal, siendo el tratamiento antibiótico correcto de extrema importancia en las primeras horas de aparición de la sintomatología. A pesar de una correcta terapia antibiótica, un tercio de los pacientes con listeremia mueren, sobretodo aquellos con enfermedades subyacentes, edad avanzada, inmunodepresión severa y sepsis asociada a afectación de sistema nervioso central. En pacientes con meningoencefalitis, la mortalidad oscila entre 38 y el $62 \%$ (8). La recidiva se estima en un $7 \%$ de los episodios $(10,11)$. En el líquido cefalorraquídeo de pacientes con meningitis por Listeria se suelen encontrar leucocitosis con predominio polimorfonuclear, particularmente si la presentación es aguda, o linfocitario si la presentación es subaguda (11) acompañado de hipoglucorraquia e hiperproteinorraquia.

La Listeria monocytogenes es sensible a un amplio grupo de antibióticos exceptuando las nuevas cefalosporinas y la fosfomicina $(12,13)$. La penicilina y la ampicilina son efectivas en el tratamiento de la listeremia. Se recomienda la asociación de un aminoglucósido, particularmente gentamicina, netilmicina o amikacina, que son igualmente efectivos (14) y aumentan la acción bactericida de la ampicilina, que sola o en combinación con gentamicina parece ser el tratamiento de elección (15).

La asociación trimetropin-sulfametoxazol ha sido considerada de segunda elección (14).

La efectividad del tratamiento antibiótico depende en gran medida de la dosis del mismo. Son necesarias altas dosis de ampicilina, sobretodo cuando hay afectación cerebral, ya que ésta cruza la barrera hemato-encefálica de una manera muy pobre, siendo necesario en adultos dosis no menores a 6 g/día (14).

La duración del tratamiento es crucial. En pacientes con afectación del sistema nervioso central se recomiendan periodos no menores a 5-6 semanas de ampicilina y 2-4 semanas de gentamicina $(10,16)$.

La asociación de listeremia y LES ha sido publicada en raras ocasiones en pacientes con (17-19) y sin tratamiento esteroideo o citostático (20). Parece que existen varios factores que predisponen a los pacientes con lupus a la infección por Listeria monocytogenes. En primer lugar, el tratamiento con corticoides y/o citostáticos, de uso extendido en pacientes con enfermedades del tejido conectivo, en segundo lugar, la frecuente asociación de insuficiencia renal o tratamiento renal sustitutivo mediante diálisis o trasplante, y por último el defecto en los linfocitos $\mathrm{T}$ y en la opsonización que presentan los pacientes con LES (21), que les hace más susceptibles a infecciones por Listeria. Evidencias experimentales indican que los linfocitos $\mathrm{T}$, sobretodo los CD8, juegan un importante papel interrumpiendo el ciclo de replicación intracelular de la Listeria (22,23). Así, en pacientes con LES que presenten fiebre con afectación neurológica hay que tener en cuenta una posible listeremia e instaurar tratamiento con ampicilina lo más pronto posible, ya que de ello dependerá en gran medida la buena evolución del cuadro. 


\section{Bibliografía}

1. Tse KC, Li FK, Chan TM, Lai KN. Listeria monocytogenes peritonitis complicated by septic shock in a patient on continuous ambulatory peritoneal dialysis. Clin Nephrol 2003; 60: 61-62.

2. Alcoba Leza M, Carro Fernández JA, Pérez Simón MR, Guerra Laso J, Alonso Fernandez A, Arto Millán L. Listeria monocytogenes meningitis in the adult in Spain. Report of 10 cases and review of the literature. Rev Clin Esp 2002; 202: 638-643.

3. Cherubin CE, Appleman MD, Heseltine PN, Khayr W, Stratton CW. Epidemiological spectrum and current treatment of listeriosis. Rev Infect Dis 1991; 13: 1108-1114.

4. Iwarson S, Lindin-Janson G, Svensson R. Listeric meningitis in the non-compromised host. Infection 1997; 6: 204-206.

5. Arias Miranda IM, Nuno Mateo FJ, Noval Menéndez J, Fonseca Aizpuru EM, Menéndez Calderón MJ. Listeriosis in the adult. Revision of 10 cases. An Med Interna (Madrid) 2004; 21: 75-78.

6. Marrón A, Rosón B, Mascaró J, Carratalá J. Listeria monocytogenes empyema in an HIV infected patient. Thorax 1997; 52: 745-746.

7. Winslow DL, Steele-Moore L. Ventriculoperitoneal shunt infection due to Listeria monocytogenes. Clin Infect Dis 1995; 20: 1437.

8. Khayr WF, Cherubin CE, Bleck TP. Listeriosis a protean disease. Infect Dis Clin Practice 1992; 1: 291-298.

9. Hernández A, Almirante B, Pahissa A, Capdevila JA, Planes AM, Gasser I, Martínez Vázquez JM. Listeriosis in the adult. Clinical, epidemiological, and therapeutic considerations based on a series of 26 cases. Enferm Infecc Microbiol Clin 1990; 8: 414-419.

10. Mylonakis E, Hohmann EL, Calderwood SB. Central nervous system infection with Listeria monocytogenes. 33 years' experience at a general hospital and review of 776 episodes from the literature. Medicine (Baltimore) 1998; 77: 313-336

11. Cherubin CE, Marr JS, Sierra MF, Becker S. Listeria an Gram-negative Bacillari meningitis in New York city, 1972-1979. Frequent cause of meningitis in adults. Am J Med 1981; 71: 199-209, 2103-2108.

12. Hof H, Nichterlein T, Kretschmar M. Management of listeriosis.Clin
Microbiol Rev 1997; 39: 345-357.

13. Jones EM, MacGowan AP. Antimicrobial chemotherapy of human infection due to Listeria monocytogenes. Eur J Clin Microbiol Infect Dis 1995; 14: 165-175.

14. Marget W, Seeliger HP. Listeria monocytogenes infections-therapeutic possibilities and problems. Infection 1988; 16 (Supl. 2): S175177.

15. Temple ME, Nahata MC. Treatment of listeriosis. Ann Pharmacother 2000; 34: 656-661.

16. Eckburg PB, Montoya JG, Vosti KL. Brain abscess due to Listeria monocytogenes: five cases and a review of the literature. Medicine (Baltimore) 2001; 8: 223-235.

17. Harisdangkul V, Songcharoen S, Lin AC. Listerial infections in patients with systemic lupus erythematosus. South Med J 1992; 85: 957-960.

18. Soga T, Shirai A, Igarashi T, Kato K, Ishigatsubo Y, Okubo T. A case of Listeria meningitis associated with systemic lupus erythematosus. Kansenshogaku Zasshi 1994; 68: 411-415.

19. Giunta G, Piazza I. Fatal septicaemia due to Listeria monocytogenes in a patient with systemic lupus erythematosus receiving cyclosporin and high prednisone doses. Neth J Med 1992; 40: 197-199.

20. Takano M, Aoki K. A case of untreated systemic lupus erythematosus presenting with listerial meningitis. Rinsho Shinkeigaku 2001; 41: 588591

21. Hellman DB, Petri M, Whiting-O'Keffe Q. Fatal infections in systemic lupus erythematosus: the role of opportunistic organisms. Medicine (Baltimore) 1987; 66: 341-348.

22. Ladel CH, Flesch IEA, Arnoldi J, Kaufmann SHE. Studies with MHCdeficient knock-out mice reveal impact of both MHC I and MHC IIdependent $\mathrm{T}$ cell responses on Listeria monocytogenes infection. J Immunol 1994; 153: 3116-3122.

23. Gregory SH, Liu CC. CD8+ T-cellmediated response to Listeria monocytogenes taken up in the liver and replicating within hepatocytes. Immunol Rev 2000; 174: 112-122. 